\title{
Salivary cortisol in pregnant women suffering from blood-and injection phobia
}

\author{
Caroline Lilliecreutz, Elvar Theodorsson, Gunilla Sydsjö and Ann Josefsson
}

\section{Linköping University Post Print}

N.B.: When citing this work, cite the original article.

The original publication is available at www.springerlink.com:

Caroline Lilliecreutz, Elvar Theodorsson, Gunilla Sydsjö and Ann Josefsson, Salivary cortisol in pregnant women suffering from blood-and injection phobia, 2011, Archives of Women's Mental Health, (14), 5, 405-411.

http://dx.doi.org/10.1007/s00737-011-0234-2

Copyright: Springer Verlag (Germany)

http://www.springerlink.com/

Postprint available at: Linköping University Electronic Press

http://urn.kb.se/resolve?urn=urn:nbn:se:liu:diva-59744 


\title{
Salivary cortisol in pregnant women \\ suffering from blood- and injection phobia
}

\author{
by \\ Caroline Lilliecreutz MD, $\mathrm{PhD}^{*}$, Elvar Theodorsson $\mathrm{MD}, \mathrm{PhD}^{* *}$, \\ Gunilla Sydsjö PhD*, Ann Josefsson MD, PhD*
}

*Department of Clinical and Experimental Medicine, Division of Obstetrics and Gynecology

**Department of Clinical and Experimental Medicine, Division of Clinical Chemistry

Faculty of Health Sciences

University of Linköping

Correspondence:

Dr C Lilliecreutz

Division of Obstetrics and Gynecology

University Hospital

SE-581 85 Linköping, Sweden.

Tel: +46 132220 00; fax: +4613 148156; E-mail: caroline.lilliecreutz@ lio.se 


\begin{abstract}
Purpose: Stress and/or anxiety during pregnancy affect maternal and fetal well-being and can cause premature delivery and postnatal pathology in the child. Women suffering from phobias related to blood and injections are prone to high levels of stress including anxiety and sometimes panic attacks during pregnancy. Cortisol is amongst the mediators through which the neurohormonal expressions of maternal psychological factors may be transduced to the fetus. The aim was to investigate if pregnant women suffering from blood- and injection phobia have raised cortisol levels or are characterized by unusual diurnal salivary cortisol profiles compared to healthy controls.
\end{abstract}

Methods: The sample consisted of 110 pregnant women with blood- and injection phobia and 110 pregnant healthy controls. Both groups provided morning and evening saliva samples in week 25 and 36 for the assay of cortisol. In gestational week 25 when blood was drawn for the mandatory blood testing extra blood was taken to analyze corticotrophin releasing factor (CRF), adrenocorticotropic hormone (ACTH) and cortisol in serum.

Results: The diurnal decline in salivary cortisol was observed as well as increased cortisol levels during pregnancy. Pregnant women suffering from blood- and injection phobia had higher output of cortisol compared to women without the phobia $(F=6.25 \mathrm{df}=1 \mathrm{p}=0.014)$ but no marked difference in the diurnal cortisol rhythm was found between the groups.

Conclusion: Our findings indicate that untreated blood- and injection phobia during pregnancy increases cortisol concentrations. Blood- and injection phobia is treatable and cognitive behavioral therapy can be used. Women with blood- and injection phobia during pregnancy therefore need to be recognized and offered a treatment without delay in early pregnancy. 
Keywords: cortisol, anxiety, stress, pregnancy, blood-and injection phobia 


\section{Introduction}

There is ample evidence from both animal and human studies showing the importance of maternal psychological functioning during pregnancy regarding maternal and fetal well-being (Herbert 2006; Seckl and Holmes 2007; Talge et al 2007; van den Bergh et al 2005;

Weinstock 2001). Blood- and injection phobia is categorized as an anxiety disorder in DSMIV (DSM-IV, 1994) due to the symptoms of anxiety and the potential panic attacks that are associated with the phobia. Classical symptoms of blood- and injection phobia is fear and avoidance of receiving injections, an aversion to seeing blood, and fear of having a blood sample taken (Hamilton 1995). There is a high probability that the person will faint or be sick when the phobic situation encountered offers no possibility to escape or to avoid the stimuli (Olatunji et al. 2006). Blood- and injection phobia is a common specific phobia with a prevalence of $7 \%$ among pregnant women (Lilliecreutz and Josefsson 2008). Its etiology is most likely a combination of genetic factors and conditioning experiences (Davey et al. 1993; Menzies and Clark 1995).

\section{The hypothalamic-pituitary-adrenocortical axis in pregnancy}

The hypothalamic-pituitary-adrenocortical (HPA) axis controls a distinctive diurnal pattern of cortisol output. The cortisol awakening response (CAR) seems to be under a separate central control (Fries et al. 2008) and there is a substantial increase in cortisol secretion after the moment of awakening (Clow et al. 2004). There is normally a decrease throughout the day reaching its nadir in late evening. The individual diurnal rhythm of cortisol is normally maintained during pregnancy (de Weerth and Buitelaar 2005).

During pregnancy there is an increased stimulation of cortisol output due to the production of corticotrophin releasing factor (CRF) produced by the placenta (Kammerer et al. 2006). There is usually a rapid return to non-pregnant levels after delivery but the normalization of the functions of the HPA axis can, however, sometimes take weeks (Mastorkos and Ilias 2003). 
Bioavailable cortisol remains at non-pregnant levels until around the $25^{\text {th }}$ week of gestation (Allolio et al. 1990) and the maternal HPA-axis becomes hypo-responsive to stress as gestation increases (Kammerer et al. 2002; Schulte et al. 1990).

Different patterns of derangement of the HPA axis regulation have been shown during situations of chronic or prolonged stress, maternal antenatal anxiety, and have also been associated with different psychopathologies (Diego et al. 2006; Gold and Chrousos 2002; Kammerer et al. 2006; Nater et al. 2008; Obel et al. 2005; Roberts et al. 2004; Sarkar et al. 2006; Taylor et al. 2009; Wessa et al. 2006).

The diurnal rhythm and/or the levels of cortisol are important indicators of the influence of maternal psychological functioning of the HPA axis. There is support in the literature that excess of glucocorticoids during fetal development exert multiple adverse effects. These include malformation of parts of the brain (e.g. the cerebellum), increased activity of the HPA axis in adult life, and increased likelihood of corticoid sensitive disorders such as hypertension, increased anxiety and exaggerated stress responses (Emack et al . 2008; Herbert et al. 2006). Rodent studies have shown that maternal prenatal stress can affect the function of the placenta, including the expression and activity of 11 beta-hydroxysteroid dehydrogenase 2 (11ß-HSD2) (Mairesse et al. 2007; Welberg et al. 2005). This enzyme metabolizes cortisol to the inactive cortisone in the placenta, and thus regulates maternal to fetal transfer. Low levels of $11 \beta-H S D 2$ in the placenta have been associated with intrauterine growth restriction, possibly due to increased fetal exposure to cortisol (Dy et al. 2008; Kajantie et al. 2003; Mc Ternan et al. 2001).

Corticosteroids act on the neurons in the brain either as a result of binding to two categories of cytoplasmic steroid receptors or by interaction with membrane-bound receptors that mediate actions of neurotransmitters such as gamma-aminobutyricacid (GABA) or glutamate. The cytoplasmic receptors have different distributions in the brain and different affinity for 
glucocorticoids. Salaria et al. showed in an in vitro model using microarray analysis on human fetal brain aggregates that increased cortisol exposure affects the expression of over a thousand genes (Salaria et al. 2006).

Cortisol is present in saliva mainly in non-protein form, representing the free, biologically active fraction $(5-10 \%)$ of the total plasma cortisol concentration. It has been suggested that salivary cortisol assesses the HPA function more appropriately than serum cortisol since cortisol in the saliva reflects the biologically active hormone (Kirschbaum and Hellhammer, 1994).

Several studies on stress and anxiety during pregnancy has described higher plasma CRH, ACTH and cortisol concentrations particular if the stress was of chronic rather than episodic nature (Demyttenaere et al.1989; Wadhwa et al. 1996; Hobel et al. 1999). Gestational stress does not only activate the maternal HPA axis but can also cause an increase in the release of CRH from the placenta by catecholamines and cortisol (Petraglia 1996). Another study by Petraglia found no relationship between psychosocial stress and CRH in gestational week 28 (Petraglia 2001). On the other hand; Sarkar et al showed that maternal state anxiety in women awaiting amniocentesis is positively correlated with plasma cortisol independent of gestation and time of collection (Sarkar 2006).

To our knowledge there are no studies investigating the diurnal rhythm of cortisol in an anxiety disorder such as a specific phobia. The current study was therefore designed to investigate whether pregnant women suffering from blood- and injection phobia have higher levels and different pattern of diurnal cortisol compared with the pregnant controls. The second aim was to investigate if the morning values of CRF, ACTH and serum-cortisol in gestational week 25 were higher among pregnant women with blood-and injection phobia comparing to the controls. 


\section{Methods}

\section{Setting and participants}

In 2005, 1606 pregnant women consequently registered at the antenatal care clinics in the south-east health district in Sweden were asked to complete the "Injection Phobia-Anxiety" scale (IPSA) (Öst et al. 1992) assessing phobic symptoms. The scale consists of 18 items describing various injection situations. The woman rates the degree of anxiety in situations involving injections from $0-4(\min 0-\max 72)$. Some examples of items included in the scale are: "Giving a blood sample by having a finger pricked, "Having a shot in the upper arm" and "Having a vein puncture". The internal consistency of the scale has been found reliable (Cronbachs alpha 0.86) (Öst et al. 1992). Amongst the participants $4.8 \%$ declined participation. 347 women scored $\geq 20$ on the" Injection Phobia-Anxiety" scale and were interviewed by telephone by one authorized psychotherapist. 110 women fulfilled the criteria for blood-and injection phobia according to DSM-IV and were invited to take part in the study, together with a randomly stratified sample of 110 women who scored $<20$ on the IPSA. The randomization was done by a research assistant who, from a data sheet, randomly picked one control woman for each index woman among the feasible group of women considering age and parity. Exclusion criteria for all subjects were being under 18 of age. The IPSA has a sensitivity of approximately $100 \%$ at threshold 20 for blood-and injection phobia (Lilliecreutz and Josefsson 2008). In order to verify that women scoring <20 on the IPSA do not suffer from blood- and injection phobia, 25 women randomly chosen in that particular group, were interviewed by telephone by the same psychotherapist, none fulfilled the criteria according to DSM-IV for blood- and injection phobia (Lilliecreutz and Josefsson 2008). All data about the study population were registered in the standardized Swedish antenatal records and are thus prospectively collected. The data were extracted from the records by the first author, The following data were collected to receive information concerning potential 
confounding variables: age, parity, marital status, occupation or smoking. Any history both past and current of psychiatric disorder (depression, anxiety disorder except blood- and injection phobia, eating disorder and psychotic disease), actual chronic medical diseases (e.g. asthma with continuous need of pharmacological treatment, epilepsy, hypertension, rheumatic arthritis, inflammatory bowel disease and diabetes) or use of antidepressants. Table 1 presents the characteristics of the study population.

Table 1. The characteristics of the study population who at least has one morning salivary cortisol in week 25 or 36

\begin{tabular}{|c|c|c|c|c|c|}
\hline & \multicolumn{2}{|c|}{$\begin{array}{l}\text { Pregnant women with } \\
\text { blood-and injection phobia }\end{array}$} & \multicolumn{2}{|c|}{$\begin{array}{l}\text { Healthy pregnant } \\
\text { controls }\end{array}$} & \multirow[t]{2}{*}{ P-value } \\
\hline & $\mathrm{N}$ & $\%$ & $\mathrm{~N}$ & $\%$ & \\
\hline \multicolumn{6}{|l|}{ Age } \\
\hline Mean, SD & 32.35 & 4.18 & 31.32 & 4.56 & 0.248 \\
\hline $16-24$ & 4 & 6.1 & 1 & 2.7 & 0.335 \\
\hline $25-34$ & 50 & 75.8 & 25 & 67.6 & \\
\hline $35-46$ & 12 & 18.2 & 11 & 29.7 & \\
\hline \multicolumn{6}{|l|}{ Parity* } \\
\hline Primi & 35 & 53.8 & 21 & 56.8 & 0.776 \\
\hline Multi & 30 & 46.2 & 16 & 43.2 & \\
\hline \multicolumn{6}{|l|}{ Marital status } \\
\hline Married/cohabiting & 65 & 98.5 & 37 & 100 & 0.452 \\
\hline Single & 1 & 1.5 & 0 & 0 & \\
\hline \multicolumn{6}{|l|}{ Smoking** } \\
\hline No & 58 & 87.9 & 37 & 100 & $0.024 *$ \\
\hline Yes & 8 & 12.1 & 0 & 0 & \\
\hline
\end{tabular}

Study design

In total 220 pregnant women were included in the study, 110 in the phobia group and 110 controls. The study was supervised by each woman's midwife at the antenatal care clinic, who administered the instructions for home saliva collection together with the material to the 
woman in the designated gestational week. The woman were asked to provide morning and evening samples for measurement of salivary-cortisol in pregnancy week 25 and 36 as we wanted to measure the sensitivity for stress and anxiety on the HPA-axis both in mid- and late pregnancy. Samples were collected immediately upon awakening and in the evening before going to bed. They were instructed not to brush their teeth, nor eat, nor drink for at least 30 min before collecting the samples. Sample collection was preferably done on ordinary days avoid of extra psychological or physical stress. The use of filter strips for saliva collection and their validation have been described in detail previously (Neu et al. 2007). Salivary samples were collected in Salivette test tubes (Sarstedt, Nuembrecht, Germany) and stored at $-20^{\circ} \mathrm{C}$. A commercial enzyme immunoassay (Salimetrics http://www.salimetrics.com) designed for the analysis of salivary cortisol was used. The lowest concentration of cortisol that can be distinguished from 0 was $<0.083 \mathrm{nmol} / \mathrm{L}$. In week 25 when the mandatory blood sample for blood testing was taken, sample for serum cortisol was collected. In addition blood was drawn for analysis of CRF and ACTH in women living close to the laboratory $(n=38$ women with blood-and injection phobia and $n=28$ controls). Blood samples were drawn from a superficial cubital vein using the closed Vacutainer system and serum collected for measurement of cortisol, ACTH and CRF. Cortisol was measured using a chemoluminiscence kit from Siemens Medical Solutions Diagnostics on an Advia Centaur XP. The lowest detectable concentration was $5.5 \mathrm{nmol} / \mathrm{L}$. ACTH was measured a chemoluminscence kit from Siemens Medical Solutions Diagnostics on an Immunlite 2500 Immuno Chemistry System. The lowest detectable concentration was $5 \mathrm{ng} / \mathrm{L}$. $\mathrm{CRF}$ was measured using a competitive radioimmunoassay in plasma extracted on $\mathrm{C}_{18}$-reverse phase columns. CRF antiserum B3-23 against human CRF (http://www.eurodiagnostica.com) and ${ }^{125} \mathrm{I}-\mathrm{CRF}-\mathrm{Tyr} \mathrm{O}^{\mathrm{o}-\mathrm{CRF}}$ (http://www.eurodiagnostica.com) was used as antiserum and 
radioligand, respectively. Human CRF SC060 (http://www.neomps.com) was used as calibrator. The lowest detectable concentration was $5 \mathrm{pmol} / \mathrm{L}$. 


\section{Statistical methods}

All statistical analyses were performed using SPSS (version 17, Chicago, Illinois, USA). Wilcoxon's non parametric test for independent variables was used when calculating for differences between serum cortisol, ACTH and CRF between the groups. Medians are used to show central tendency and inter-quartile range to illustrate variation. The salivary cortisol measurements were not normally distributed. Hence all measurements were transformed by natural logarithms in order to achieve normality which was verified using the Kolmogorov-Smirnov test. All further parametric inferential statistics were performed on these transformed variables. Dixon test for outliers confirmed exclusion of two morning values in the control group; one in week $25(43.6 \mathrm{nmol} / \mathrm{L})$ and one in week $36(49.7$ $\mathrm{nmol} / \mathrm{L})$.

Repeated measures ANOVA with the measurements of cortisol levels at the different occasions as within-subject and phobic status, gestational week, age and parity as betweensubject were performed in order to detect within-group as well as between group differences over time.

Pearson's correlation was used both to explore the magnitude and direction of the linear relationship between morning salivary cortisol levels and birth weight and between morning salivary cortisol and serum cortisol in gestational week 25 .

\section{Ethics}

The study protocol was approved by the ethics committee of the University Hospital in Linköping, Sweden. 


\section{Results}

The individuals in the study population who had both morning and evening cortisol values either in one or both weeks were included in the analysis of salivary cortisol.

In a repeated measurement ANOVA with cortisol levels at the different occasions as withinsubject and age, parity, gestational week and phobic status as between-subject the women with blood- and injection phobia had higher levels of cortisol comparing to the healthy controls $(\mathrm{F}=6.25, \mathrm{df}=1, \mathrm{p}=0.014)$. If smoking was included as a between-subject variable the effect remained $(\mathrm{F}=6.26, \mathrm{df}=1, \mathrm{p}=0.013)$. Table 2 presents descriptive statistics and final sample sizes.

Table 2 Maternal diurnal salivary cortisol levels (08 am 3.5-27 nmol/L $10 \mathrm{pm}<6.0 \mathrm{nmol} / \mathrm{L}$ in a non pregnant population)

Gestational week Morning (M) Evening (E)
Pregnant women with Healthy pregnant controls blood-and injection phobia

\begin{tabular}{|l|l|l|l|l|l|l|l|l|}
\hline & $\mathrm{N}$ & $25^{\text {th }}$ & Median & $75^{\text {th }}$ & $\mathrm{N}$ & $25^{\text {th }}$ & Median & $75^{\text {th }}$ \\
\hline $25 \mathrm{M}$ & 65 & 9.5 & 12.7 & 16.6 & 37 & 10.3 & 12.6 & 15.4 \\
\hline $25 \mathrm{E}$ & 65 & 2.0 & 2.9 & 3.9 & 35 & 1.7 & 2.6 & 3.2 \\
\hline $36 \mathrm{M}$ & 42 & 10.8 & 13.8 & 16.9 & 21 & 9.5 & 13.8 & 16.3 \\
\hline $36 \mathrm{E}$ & 43 & 3.1 & 4.0 & 5.4 & 21 & 3.0 & 3.9 & 5.0 \\
\hline
\end{tabular}

This ANOVA also showed that the cortisol levels concerning the whole study population in gestational week 36 (Mean=9.1, SD=5.9) was higher compared to gestational week 25 (Mean 8.3, $\mathrm{SD}=6.6, \mathrm{~F}=7.27, \mathrm{df}=1, \mathrm{p}=0.008)$. The diurnal decline in salivary cortisol was observed in both groups $(\mathrm{F}=60.48, \mathrm{df}=1, \mathrm{p}<0.000)$.

The ANOVA model also tested for interactions between cortisol levels and gestational week $(\mathrm{F}=0.211, \mathrm{df}=1, \mathrm{p}=0.65)$, parity $(\mathrm{F}=0.80, \mathrm{df}=1, \mathrm{p}=0.37)$ and age $(\mathrm{F}=2.30, \mathrm{df}=2, \mathrm{p}=0.10)$ 
between the women with blood- and injection phobia and the healthy controls. However, no statistically significant interactions were found.

The nonparametric Wilcoxon's test was used to test for differences in serum concentrations of CRF, ACTH and cortisol in serum in the two groups. No statistically significant differences were found (Table 3).

Table 3. Maternal corticotrophin releasing hormone (fP-CRH ref $<5 \mathrm{pmol} / \mathrm{L}$ ), adrenocorticotropin hormone (P-ACTH ref <46ng/L) and serum cortisol (S-cortisol 07am 200-700 $\mathrm{nmol} / \mathrm{L}$ ). The reference values are for a non-pregnant population

\begin{tabular}{|l|l|l|l|l|l|l|l|l|}
\hline & $\mathrm{N}$ & $25^{\text {th }}$ & Median & $75^{\text {th }}$ & $\mathrm{N}$ & $25^{\text {th }}$ & Median & $75^{\text {th }}$ \\
\hline Cortisol & 73 & 555.8 & 678.1 & 766.4 & 53 & 539.5 & 631.3 & 728.9 \\
\hline ACTH & 15 & 14.0 & 24.3 & 35.6 & 17 & 11.6 & 17.6 & 36.0 \\
\hline CRH & 21 & 1.2 & 4.4 & 8.1 & 22 & 2.5 & 5.3 & 10.7 \\
\hline
\end{tabular}

There were no significant correlations between morning salivary cortisol levels (week 25, r=0.024, $\mathrm{p}=0.82$ week $36, \mathrm{r}=-0.031, \mathrm{p}=0.81$ ) and birth weight. A correlation between morning salivary cortisol and serum cortisol in gestational week 25 ( $r=0.208, p=0.042$ ) was found. Women with blood- and injection phobia were over-represented in the lower socioeconomic groups ( $\mathrm{p}=0.018$ ), The absolute numbers of individuals in the study population concerning the variables smoking and psychiatric disorder was low. Only one individual in the group with blood-and injection phobia was using antidepressant and none in the control group. 


\section{Discussion}

This study shows that the pattern of the diurnal secretion of cortisol in pregnant women suffering from blood- and injection phobia did not differ from that of women without the phobia.

However, women with blood- and injection phobia seem to have higher levels of cortisol output compared to the control group. This finding has an important clinical implication as these patients are common at the antenatal care clinics and studies have shown adverse obstetric and neonatal outcomes for these patients. Pregnant women with blood- and injection phobia are more likely to be delivered by elective cesarean section and having a baby born with a complication such as small for gestational age and prematurity (Lilliecreutz and Josefsson 2008; Lilliecreutz et al. 2010)

Elevated levels of cortisol due to stress and anxiety can alter the obstetric and neonatal outcome resulting in premature labor, shortened pregnancy length, low-birth weight and impaired fetal brain development (Copper 1996; Field, 2006; Wadhwa 1993; Weinstock, 2005).

Studies of samples from non-pregnant women have shown afternoon cortisol levels to be the most susceptible parameter to the influence of psychological stress (Grossi et al. 2001; Powell et al. 2002). We were unable to confirm this finding in our study of pregnant women indicating that pregnancy- induced resetting of the HPA axis blurs the relation between stress and cortisol levels in the evening.

The maternal HPA axis normally becomes hypo-responsive to stress as gestation progresses and previous research has shown that maternal psychological functions only marginally impact the diurnal rhythm of cortisol in late pregnancy (Kammerer et al. 2002; Petraglia et al. 2001; Schulte et al.1990; Urizar et al. 2004), which our current results corroborate. 
It is likely that the HPA axis in nulliparas is more responsive than in women which have given birth at least once. It has been suggested in some studies that nulliparas have lower waking cortisol levels compared to multiparous women and that the midday cortisol levels are higher compared to multiparous women (Jones et al. 2006; Rasheed et al. 1993; Vleugels et al.1986). On the other hand, Goedhart et al. have shown that women with higher age and parity have lower maternal cortisol levels in the morning but that study was performed in the first trimester (Goedhart et al. 2009). We could not find any interactions in our study between cortisol levels, age and parity.

Other psychiatric disorders have been associated with altered cortisol levels; melancholic depression is associated with raised cortisol and atypical depression with reduced cortisol output (Gold and Chrousos 2002). Posttraumatic stress disorder (PTSD) as well as chronic fatigue syndrome have been reported to show a blunted CAR (Nater et al. 2008; Roberts et al. 2004; Wessa et al. 2006). Postnatal depressive symptoms are associated with major changes in the function of the HPA axis after delivery and have shown to have higher cortisol levels at waking and no increase at +30 min compared to controls (Kammerer et al. 2006; Taylor et al. 2009).

One limitation in our study is the missing cortisol values at one or more collection points. These included failure to collect as directed which has its roots in the patient material and study design. Nevertheless women in the current study displayed the expected pattern of salivary cortisol decline across the day and the mean salivary cortisol levels were similar to those reported by others for women in their $36^{\text {th }}$ week of pregnancy (Allolio et al. 1990; Kivlighan et al. 2008; Meulenberg and Hofman 1990) and approximately 1.5 times higher than mean values reported for non-pregnant controls. These observations confirm that women in this sample displayed the moderate elevations in salivary cortisol normal for late pregnancy. 
The support for an association between placental weight and birth weight is well-recognized in the literature (Jansson and Powell 2007). However, Kivlighan and colleagues also report an inverse association between morning cortisol levels, placental weight and birth weight (Kivlighan et al. 2008). We could not confirm this result in our study as no correlation was found between morning salivary cortisol levels and birth weight, which is in line with a recently published study by Goedhart (Goedhart et al. 2009).

Amongst the strengths of the present study are the population-based design, the diagnoses based on clinical interviews instead of questionnaires, and the information available on an individual basis on most of the plausible confounding factors (age, parity and gestational week) for the association between blood- and injection phobia and a change in the levels and diurnal pattern of cortisol which were controlled for in the analyses, providing robust results. Finally Fleshner and colleagues (Fleshner et al. 1995) suggested that stress decreases the corticotrophin-binding globulin (CBG) levels resulting in increased levels of unbound cortisol and it is therefore preferable to measure cortisol in saliva and not in serum.

Earlier clinical and experimental studies have shown the detrimental effects of conditions associated with increasing cortisol concentrations in pregnancy. Our findings indicate that untreated blood- and injection phobia during pregnancy increases cortisol concentrations. The women with blood- and injection phobia during pregnancy therefore need to be recognized and offered a treatment without delay in early pregnancy. 


\section{References}

Allolio DD, Hoffmann J, Linton EA, WinklmannW, Kusche M, Sculte HM (1990) Diurnal salivary cortisol patterns during pregnancy and after delivery: relationship to plasma corticotrophin-releasing-hormone. Clin Endocrinol (Oxf.) 33:279-289.

Clow A, Thorn L, Evans P, Hucklebridge F (2004) The awakening cortisol response: methodological issues and significance. Stress 7:29-37.

Copper RL, Goldenberg RL, Das A, Elder N, Swain M, Norman G, Ramsey R, Cotroneo P, Collins BA, Johnson F, Jones P, Meier AM (1996) The preterm prediction study: maternal stress is associated with spontaneous preterm birth at less than thirty-five weeks' gestation. National Institute of Child Health and Human Development Maternal-Fetal Medicine Units Network. Am J Obstet Gynecol 175:1286-1292.

Davey GC, De Jong PJ, TallisF (1993) UCS inflation in the aetiology of a variety of anxiety disorders: some case histories. Behav Res Ther 31:495-498.

Demyttenaere K, Nijs P, Evers-Kiebooms G, Koninckx PR (1989)The effect of a specific emotional stressor on prolactin, cortisol, and testosterone concentrations in women varies with their trait anxiety. Fertil Steril. 52:942-948.

de Weerth C, Buitelaar JK (2005) Cortisol awakening response in pregnant women. Psychoneuroendocrinology 30: 902-907.

Diego MA, Jones NA, Field T, Hernandez-Reif M, Schanberg S, Kuhn C et al (2006) Maternal psychological distress, prenatal cortisol, and fetal weight. Psychosom Med 68:747753.

DSM-IV: Diagnostic and Statistic Manual of Mental Disorders, fourth edition, American Psychiatric Association (1994)

Dy J, Guan H, Sampath-Kumar R, Richarsoon BS, Yang K (2008) Placental 11 betahydroxysteroid dehydrogenase type 2 is reduced in pregnancies complicated with idiopathic intrauterine growth restriction: evidence that this is associated with an attenuated ratio of cortisone to cortisol in the umbilical artery. Placenta 29:193-200.

Emack J, Kostaki A, Walker CD, Matthews SG (2008) Chronic maternal stress affects growth, behaviour and hypothalamic-pituitary-adrenal function in juvenile offspring. Horm Behav 54: 514-520.

Field T, Hernandez-Reif M, Diego M, Figueiredo B, Schanberg S, Kuhn C (2006) Prenatal cortisol, prematurity and low birthweight. Infant Behav Dev 29:268-275.

Fleshner M, Deak T, Spencer RL, Laudenslager ML, Watkins LR, Maier SF (1995) A longterm increase in basal levels of corticosterone and a decrease in corticosteroid-binding globulin after acute stressor exposure. Endocrinology 136:5336-5342. 
Fries E, Dettenborn L, Kirschbaum C (2008) The cortisol awakening response (CAR): Fact and future directions. Int J Psychophysiol 72:67-73.

Goedhart G, Vrijkotte TGM, Roseboom TJ, van der Wal M.F, Cuijpers P, Bomsel GJ (2010) Maternal cortisol and offspring birthweight: Results from a large prospective cohort study. Psychoneuroendocrinology 35:644-52.

Gold PW, Chrousos GP (2002) Organization of the stress system and its dysregulation in melancholic and atypical depression: high vs low CRH/NE status. Mol Psychiatry 7:254-275.

Grossi G, Perski A, Lundberg U, Soares J (2001) Associations between financial strain and the diurnal salivary cortisol secretion of long-term unemployed individuals. Integr Physiol Behav Sci 36:205-219.

Hamilton JG (1995) Needle phobia: a neglected diagnosis. J Fam Pract 41:169-175.

Herbert J, Goodyer IM, Grossman AB, Hastings MH, de Kloet ER, Lightman SL, Lupien SJ, Roozendaal B, Seckl JR (2006) Do corticosteroids damage the brain? J Neuroendocrinol 18:393-411.

Hobel C, Dunkel-Schetter C, Roesch S, Castro L, Arora C (1999) Maternal plasma corticotropin-releasing hormone associated with stress at 20 weeks' gestation in pregnancies ending in preterm delivery. Am J Obstet Gynecol 180: 257-263.

Jansson T, Powell TL (2007) Role of the placenta in fetal programming: underlying mechanisms and potential interventional approaches. Clin Sci 113:1-13.

Jones NM, Holzman CB, Zanella AJ, Leece CM, Rahbar MH (2006) Assessing mid-trimester salivary cortisol levels across three consecutive days in pregnant women using an at-home collection protocol. Paediatr Perinat Epidemiol. 20:425-37.

Kajantie E, Dunkel L, Turpeinen U, Stenman UH, Wood PJ, Nuutila M et al. (2003) Placental 11 beta-hydroxysteroid dehydrogenase 2 and fetal cortisol/cortisone shuttle in small preterm infants. J.Clin.Endocrinol. Metab 88:493-500.

Kammerer M, Adams D, von Castelberg B, Glover V (2002) Pregnant women become insensitive to cold stress. BMC Pregnancy Childbirth 2: 8.

Kammerer M, Taylor A, Glover V (2006) The HPA axis and perinatal depression: a hypothesis. Arch Womens Ment Health 9:187-196.

KirschbaumC, Hellhammer DH (1994). Salivary cortisol in psychoneuroendocrine research: recent developments and applications. Psychoneuroendocrinology 19:313-333.

Kivlighan KT, DiPetroJA, Costigan KA, Laudenslanger ML (2008) Diurnal rhythm of cortisol during late pregnancy: associations with maternal psychological wellbeing and fetal growth. Psychoneuroendocrinology 33:1225-1235. 
Lilliecreutz C, Josefsson A ( 2008) Prevalence of blood- and injection phobia among pregnant women. Acta Obstet Gynecol Scand 87:1276-1279.

Lilliecreutz C, Sydsjö G, Josefsson A (2010) Obstetric and perinatal outcomes among women with blood-and injection phobia during pregnancy. Accepted for publication in $\mathrm{J}$ Affect Disord.

Mairesse J, LesageJ, BretonC, BreantB, HahnT, Darnaudery M (2007) Maternal stress alters endocrine function of the feto-placental unit in rats. Am. J. Physiol. Endocrinol Metab 292: E1526-E1533.

Mastorakos G, Ilias I (2003) Maternal and Fetal Hypothalamic-Pituitary-Adrenal Axes During Pregnancy and Postpartum. Ann.N.Y.Acad.Sci. 997:136-149.

Mc Ternan CL, Draper N, Nicholson H, Chalder SM, Driver P, Hewison M et al (2001). Reduced placental 11 beta-hydroxysteroid dehydrogenase type 2 mRNA levels in human pregnancies complicated by interuterine growth restriction: an analysis of possible mechanisms. J Clin Endocrinol Metab 86:4979-4983.

Menzies RG, Clark JC (1995) The aetiology of phobias: a non-associative account. Clin Psychol Rev. 5:23-48.

Meulenberg PM, Hofman JA (1990) The effect of oral contraceptive use and pregnancy on the daily rhythm of cortisol and cortisone. Clin Chem Acta 190:211-221.

Nater UM, Youngblood L-S, Jones J.F, Unger ER, Miller AH, Reeves WC et al (2008). Alterations in diurnal salivary cortisol rhythm in a population-based sample of cases with chronic fatigue syndrome. Psychosom.Med 70:298-305.

Neu M, Goldstein M., Gao D, Laudenslager ML (2007) Salivary cortisol in preterm infants: validation of a simple method for collecting saliva for cortisol determination. Early Hum Dev 83: 47-54.

Obel C, Hedegaard M, Henriksen TB, Secher NJ, Olsen J, Levine S (2005) Stress and salivary cortisol during pregnancy. Psychoneuroendocrinology.30:647-656.

Olatunji BO, Williams NL, Sawchuk CN, LohrJM (2006) Disgust, anxiety and fainting symptoms associated with blood-injection-injury fears: a structural model. J Anxiety Disord 20:23-41.

Petraglia F, Florio P, Nappi C, Genazzani AR (1996) Peptide signaling in human placenta and membranes: autocrine, paracrine, and endocrine mechanisms. Endocr Rev.17:156-186.

Petraglia F, Hatch MC, Lapinski R, Stomati M, Reis FM, Cobellis L et al( 2001) Lack of effect of psychosocial stress on maternal corticotrophin-releasing factor and catecholamine levels at 28 weeks gestation. J Soc Gynecol Invest 8:83-88.

Powell LH, Lovallo WR, Matthews KA, Meyer P, Midgley AR, Baum A (2002) Physiologic markers of chronic stress in premenopausal, middle-aged women. Psychosom Med 64:502509. 
Rasheed FN(1993) Parity, birthweight, and cortisol. Lancet. 341:828.

Roberts AD, WesselyS, ChalderT, Papadopoulos A, Cleare AJ (2004) Salivary cortisol response to awakening in chronic fatigue syndrome. Br J Psychiatry 184:136-141.

Salaria S, ChanaG, Caldara F, Feltrin E, Alteri M, Faggioni F et al (2006) Microarray analysis of cultured human brain aggregates following cortisol exposure: implications for cellular functions relevant to mood disorders. Nerobiol Dis 23:630-636.

Sarkar P, BergmanK, Fisk NM, GloverV (2006) Maternal anxiety at amniocentesis and plasma cortisol. Prenat Diagn 26:505-509.

Schulte HM, Weisner D, Allolio B (1990) The corticothrophin releasing hormone test in late pregnancy: lack of adrenocorticothrophin and cortisol response. Clin Endocrinol (Oxf.). 33: 99-106.

Seckl JR, Holmes MC (2007) Mechanisms of disease: glucocorticoids, their placental metabolism and fetal programming of adult pathophysiology. Nat Clin Pract Endocrinol Metab 3: 479- 488.

Talge NM Neal,C, Glover V (2007) Antenatal maternal stress and long-term effects on child neurodevelopment:how and why? J. Child Psychol. Psychiatry 48:245-261.

Taylor A, Glover V, Marks M, Kammerer M ( 2009) Diurnal pattern of cortisol output in postnatal depression. Psychoneuroendocrinology 34:1184-1188.

Urizar GG, Milazzo M, Le H-N, Delucchi K, Sotelo R, Munoz RF (2004) Impact of stress reduction instructions on stress and cortisol levels during pregnancy. Biol Psychol 67: 275282.

Van den Bergh BR, Mulder EJ, Mennes M, Glover V (2005) Antenatal maternal anxiety and stress and the neurobehavioural development of the fetus and child: links and possible mechanisms. A review. Neurosci Biobehav Rev 29:237-258.

Vleugels MP, Eling WM, Rolland R, de Graaf R (1986) Cortisol levels in human pregnancy in relation to parity and age. Am J Obstet Gynecol. 155:118-21

Wadhwa PD, Sandman CA, Porto M, Dunkel-Schetter C, Garite TJ (1993) The association between prenatal stress and infant birth weight and gestational age at birth: a prospective investigation. Am J Obstet Gynecol 169:858-865.

Wadhwa PD, Dunkel-Schetter C, Chicz-Demet A, Porto M, Sandman CA.(1996) Prenatal psychosocial factors and the neuroendocrine axis in human pregnancy. Psychosomatic Medicine 58: 432-446.

Weinstock M (2001) Alterations induced by gestational stress in brain morphology and behaviour of the offspring. Prog Neurobiol 65:427-451.

Weinstock M. (2005) The potential influence of maternal stress hormones on development and mental health of the offspring. Brain Behav Immun 19:296-308. 
WelbergLA, Thrivikraman KV, Plotsky PM (2005) Chronic maternal stress inhibits the capacity to up-regulate placental $11 \beta$-hydroxysteroid dehydrogenase type 2 activity.

J. Endocrinol 186: R7-R12.

Wessa M, Rohleder N, Kirschbaum C, Flor H (2006) Altered cortisol awakening response in posttraumatic stress disorder. Psychoneuroendocrinology 31:209-215.

Öst L-G, Hellström K, Kåver A (1992) One versus five session of exposure in the treatment of injection phobia. Behav Res Ther 23:263-282. 